SINAI Journal of Applied Sciences 9 (1) 2020 029-040

\begin{tabular}{lll} 
Available online at www.sinjas.journals.ekb.eg & $\overline{\text { ARU-EGYPT }}$ \\
\hline SCREENED BY SINAI Journal of Applied Sciences
\end{tabular}

\title{
EFFECT OF CHEMICAL NITROGEN FERTILIZER LEVEL AND HUMIC ACID RATE ON Gladiolus grandiflorus PRODUCTIVITY
}

\author{
Hadeer R.M. El-Kot ${ }^{*}$; Sonia A. Abdallah; H.M.S. Hassan, and M.A.M. Ali
}

Dept. Plant Prod., Fac. Environ. Agric. Sci., Arish Univ., Egypt.

\begin{tabular}{l} 
ARTICLE INFO \\
\hline Article history: \\
Received: 05/01/2020 \\
Revised: $20 / 01 / 2020$ \\
Accepted: $29 / 03 / 2020$ \\
Available online: $01 / 05 / 2020$ \\
\hline Keywords: \\
Chemical N fertilization, \\
humic acid, \\
Gladiouls grandiflorus.
\end{tabular}

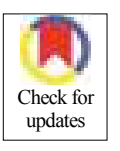

\begin{abstract}
A field experiment was conducted at the Experimental Farm, Faculty of Environmental Agricultural Science, Arish University, Egypt, during two successive seasons 2017-2018 and 2018-2019 to study the effect of various levels of nitrogen fertilizer $\left(0,100,200\right.$ and $\left.300 \mathrm{~kg} \mathrm{fed}^{-1}\right)$ in combination with different rates of humic acid $(0,150,250$ and $350 \mathrm{ppm})$ as a foliar spray on plant growth, flowering characteristics, corms and cormels characteristics and chemical constituents. The obtained results cleared that using $200 \mathrm{~kg} \mathrm{fed}^{-1}$ nitrogen in urea forms with $350 \mathrm{mgl}^{-1}$ humic acid as a foliar spray gave the maximum value in each of vegetative growth parameters, flowering characteristics, diameter of corms, weight of cormel, and chemical constituents (N, P and $\mathrm{K}$ ) content in leaves. However, a non-significant variation was observed in the treatment $200 \mathrm{~kg} \mathrm{fed}^{-1}$ nitrogen with the three concentrations of humic acid for weight of corms and No. of cormels in plants in most cases. While, plants grown without nitrogen and humic acid (control) gave the minimum values in all parameters.
\end{abstract}

\section{INTRODUCTION}

Gladiolus (gladiolus grandiflorus L.) is perennial bulbous flowering belongs to family Iridacea and subfamily Ixoidea. (Goldblatt and Manning, 1998) and Manning and Goldblatt, 2008). Gladiolus is one of the most ornamental bulbous plants grown commercially in many parts of the world for its fascinating flowers with variety of colours, huge form of florets and good keeping quality as a cut flower it has earned its place of importance owing to its utility in bouquets, vases, flower decorates and to get high market returns. Further, it may be stated that the shelf life or longevity of its flowers which is an important aspect of cut flower quality (Patra and Mohanty, 2015). Nitrogen plays an important role in building up protoplasm and protien, which induce cell division and initiate merstimatic activities when applied in optimum quality
(Singh and Ramasingh, 1969). It is one of the most important nutrients promoting yield and growth in gladiolus. The leaves should contain 2.5 to 3.0 percent or more nitrogen in dry weight for optimum yield (Bose and Yadav, 1989). Nitrogen deficiency results in pale green coloration of foliage and reduction in the number of florets spike and number of spikes, corms and also hampered the utilization of food in old reserve (Woltz 1972).

Humic acid is a natural organic polimar composition produced as a result of decaying organic matter, peat as well as lignin, and so that can be used to increase productivity and its quality (Valdrighi $\boldsymbol{e t}$ al., 1996). The positive effects are directly on the plant growth and improve the growth of shoots and roots, absorpotion of nitrogen, calcium, potassium, magnesium and phosphorus by plant (Haghighi et al., 2011). The application of humic acid

\footnotetext{
* Corresponding author: E-mail address: hadear.ramdan92@yahoo.com https://doi.org/10.21608/SINJAS.2020.86376

(C) 2020 SINAI Journal of Applied Sciences. Published by Fac. Environ. Agric. Sci., Arish Univ. All rights reserved.
} 
increase soil aggregation, structure. moisture, holding capacity, fertility and microorganisms activity (Chen and Aviad, 1990, Sharif et al., 2002).

\section{MATERIALS AND METHODS}

A field experiment was conducted at the Experimental Farm, Fac. Environ. Agric. Sci., Arish Univ., during two successive season 2017-2018 and 2018-2019 to study the effect of various levels of nitrogen fertilizer $\left(0,100,200\right.$ and $\left.300 \mathrm{~kg} \mathrm{fed}^{-1}\right)$ as urea $(46.5 \% \quad \mathrm{~N})$ in combination with different levels of humic acid (0, 150, 250 and $350 \mathrm{mgl}^{-1}$ ) on plant growth, flowering characteristics, corms and cormels characteristics and chemical constituents.

\section{Plant Materials}

The gladiolus corms were obtained from El-Ahaly Farm in El-Qanater El-Khayrea. The size of used corm was about $2.89 \mathrm{~mm}$.

Corms were planted on first of October during 2017-2018 and 2018-2019 for the two seasons in loamy sand soil of the experimental unites at $50 \times 30 \mathrm{~cm}$ distance between plants and rows, respectively. The irrigation system of the experiment was drip irrigation.

Recommended dose of super phosphate at the rate of $200 \mathrm{~kg} / \mathrm{fed}$ and potassium sulphate at the rate of $50 \mathrm{~kg} / \mathrm{fed}$ as well as chicken manure at the rate of $5 \mathrm{~kg} / \mathrm{m}^{2}$ were added to the soil before planting.

Nitrogen fertilizer in the form of urea $(46.5 \% \mathrm{~N})$ was applied in 4 levels $(0,100$, 200 and $300 \mathrm{~kg} / \mathrm{fed}$ ), This amount divided into 3 doses, The first dose was applied after month from the date of planting, the second dose applied in the stage of 2-3 leaves/plant and the last dose was applied after 2 weeks from flowering beginning.

Humic acid (as a form of humate potassium 90\%) was obtained from Grow Tech for Agricultural Development Company. Foliar spray with humic acid was applied at
4 levels $(0,150,250$ and $350 \mathrm{ppm})$. Stock solution of humic acid was prepared by dissolving the amount of humic acid powder in distilled water according to the application doses. Humic acid sprayed on the leaves of the plants three times, the first one was applied in the stage of 2-3 leaves/plant, the second one was applied in the stage of 6-7 leaves /plant and the last one was applied after spike emergence.

\section{Post Harvest Treatment}

Immediately after harvest, flowers were kept in cool water to remove the field heat. Then, were taken to the laboratory and recut again at the base and kept in vase solution composite from $200 \mathrm{ml}$ distilled water, $5 \%$ sucrose and $400 \mathrm{ppm}$ cetric acid to study the vase life of spike (Anserwadekar and Patil, 1986).

\section{Experimental Design}

This experiment included 16 treatments which were the combination between four nitrogen fertilizer levels and humic acid levels. These treatments were arranged in a split plot in randomized complete blocks design with three replicates.

\section{Data Recorded}

\section{Growth parameters}

After two months and half from corm planting and after spike emergence, the following data were recorded: number of leaves, plant height $(\mathrm{cm})$, spike length $(\mathrm{cm})$, rachis length $(\mathrm{cm})$, fresh and dry weights of leaves $(\mathrm{g})$, diameter of spike $(\mathrm{mm})$ and weight of spike $(\mathrm{g})$.

\section{Flowering characteristics}

Number of flowers per spike were taken after three months from corm planting and when first floret from the basal opening, days to spike emergence were taken after two months and half from planting the corm. Also, days to first floret opening were taken after three months from planting the corm. In addition, vase life was taken 
Table 1. The physical and chemical analyses of experiment soil during 2017/2018 and 2018/2019 seasons.

\begin{tabular}{|c|c|c|}
\hline Property & First season (2017-2018) & Second season (2018-2019) \\
\hline \multicolumn{3}{|c|}{ Particles size distribution (\%) } \\
\hline Coarse sand (\%) & 58.0 & 59.5 \\
\hline Fine sand $(\%)$ & 19.8 & 19.3 \\
\hline Silt $(\%)$ & 12.9 & 13.0 \\
\hline Clay $(\%)$ & 9.3 & 9.2 \\
\hline Soil texture & Loamy sand & Loamy sand \\
\hline Bulk density $\left(\mathrm{Mgm}^{-1}\right)$ & 1662 & 1661 \\
\hline \multicolumn{3}{|c|}{ Chemical properties (Soluble ions (in 1:5 soil water extract) } \\
\hline $\mathrm{Ca}^{+}\left(\mathrm{me}^{-1}\right)$ & 3.90 & 3.90 \\
\hline $\mathrm{Mg}^{+}\left(\mathrm{me}^{-1}\right)$ & 3.62 & 3.43 \\
\hline $\mathrm{Na}^{+}\left(\mathrm{me}^{-1}\right)$ & 2.54 & 2.59 \\
\hline $\mathbf{K}^{+}\left(\mathbf{m e}^{-1}\right)$ & 0.34 & 0.32 \\
\hline $\operatorname{CO3}^{-}\left(\mathrm{me}^{-1}\right)$ & - & - \\
\hline $\operatorname{HCO3}^{-}\left(\mathrm{me}^{-1}\right)$ & 4.30 & 4.40 \\
\hline $\mathrm{Cl}^{-}\left(\mathrm{me}^{-1}\right)$ & 4.70 & 4.35 \\
\hline $\mathrm{SO}_{4}\left(\mathrm{me}^{-1}\right)$ & 1.50 & 1.45 \\
\hline $\begin{array}{c}\mathrm{EC}\left(\mathrm{dSm}^{-1}\right) \text { in } 1: 5 \text { water } \\
\text { extract })\end{array}$ & 0.08 & 1.02 \\
\hline $\begin{array}{l}\text { pH (in1: } 2.5 \text { Soil water } \\
\text { suspension extract) }\end{array}$ & 8.10 & 8.13 \\
\hline Organic matter (\%) & 0.153 & 0.171 \\
\hline $\mathrm{CaCO}_{3}(\%)$ & 22.43 & 22.48 \\
\hline
\end{tabular}

The experiment soil and irrigation water analyses were presented in Table.1 and Table.2.

Table 2. The chemical analysis of the irrigation water during 2017/2018 and 2018/2019 seasons

\begin{tabular}{|c|c|c|c|c|c|c|c|c|c|}
\hline \multirow{3}{*}{ pH } & \multirow{3}{*}{$\begin{array}{c}\text { EC } \\
\text { dSm }^{-1}\end{array}$} & \multicolumn{8}{|c|}{ Soluble ions $\left(\mathrm{me} \mathrm{I}^{-1}\right)$} \\
\hline & & \multicolumn{4}{|c|}{ Cations } & \multicolumn{4}{|c|}{ Anions } \\
\hline & & $\mathrm{Ca}^{++}$ & $\mathbf{M g}^{++}$ & $\mathrm{Na}^{+}$ & $\mathbf{K}^{+}$ & $\mathrm{Cl}^{-}$ & $\mathrm{HCO}_{3}^{-}$ & $\mathrm{CO}_{2}^{--}$ & $\mathrm{SO}_{4}^{--}$ \\
\hline \multicolumn{10}{|c|}{ First Season (2017-2018) } \\
\hline 7.55 & 5.93 & 20.50 & 16.80 & 18.50 & 0.24 & 45.92 & 2.90 & - & 7.22 \\
\hline \multicolumn{10}{|c|}{ Second Season (2018-2019) } \\
\hline 7.60 & 6.00 & 21.00 & 17.00 & 18.80 & 0.25 & 46.75 & 2.97 & - & 7.28 \\
\hline
\end{tabular}


when half number of florets per spike were dried (Khalf-Allah, 2014). Moreover, water uptake by stems was calculated by measuring the volume of water at the termination of vase life and subtracting it from the initial quantity of distilled water in jars.

\section{Corms and cormels parameters}

After two months from cutting the flowers the following data were recorded: weight of corms $(\mathrm{g})$, weight of cormels $(\mathrm{g})$, diameter of corms $(\mathrm{mm})$ and number of cormels.

\section{Chemical constituents}

The oven dried materials of leaves were ground and wet digested by a sulfuricperocloric acids and mixture as described by Peterburgski (1968). The total N, P and $\mathrm{K}$ were determined according to the methods of Pregle (1945) and Jackson (1967).

\section{Statical Analysis}

All collected data were analyzed with analysis of variance (ANOVA) producer using SPSS. Means differences were compared by using Duncan multiple range test at 0.05 level (Duncan, 1955).

\section{RESULTS AND DISCUSSION}

\section{Vegetative Growth Parameters}

Table (3) presented the effect of nitrogen fertilization as urea in combination with humic acid rate on vegetative growth of Gladiolus grandiflorus plant. Results revealed that application of $200 \mathrm{~kg} \mathrm{~N} \mathrm{fed}^{-1}$ with 350 and $250 \mathrm{mg} \mathrm{l}^{-1}$ gave high values in all vegetative growth parameters in both seasons.

Increasing nitrogen fertilization from 100 to $200 \mathrm{~kg} \mathrm{fed}^{-1}$ and humic acid from $150 \mathrm{mg}^{-1}$ to $350 \mathrm{mg}^{-1}$ influenced the gladiolus $(\mathrm{N}$. of leaves, plant height, length of spike, rachis length, diameter of spike, weight of spike, leaf fresh weight and leaf dry weight). These results are in harmony with Chaudhary et al. (2013) who proved that Rose var. Top secret plants fertilized with $300 \mathrm{mg}$ nitrogen/plant/week in the form of urea gave a maximum number of leaves. Also, Baldoto and Baldoto (2013) observed that number of leaves of gladiolus plant increased with gradually increasing humic acid levels (from 10 to $40 \mathrm{mmol}^{-1}$ ).

These results may be due to that nitrogen elements is a major component of amino acids, the building blocks of protein (Harper and Paulsem, 1969) and thereby it plays an important role in metabolic activities of the plant resulting in the synenthis of chlorophyll and cytochromes, which are essential for photosynthesis and respiration process in the plants (Thana et al., 2008). Furthermore, El-Khateeb et al. (2017) stated that application of humic acid significantly increased the growth attributes and marjordm plants including plants height, fresh weight compared to untreated plants.

\section{Flowering Characteristic}

The effect of nitrogen fertilization as urea in combination with humic acid rate on flowering characteristics was presented in Table 4.

Results showed that treatments of nitrogen with humic acid gave a significant difference on all flowering characteristics. Plants fertilized with $200 \mathrm{~kg} \mathrm{~N}$ fed $^{-1}$ and sprayed with 0 and $150 \mathrm{ppm}$ humic acid sprouted the spike early and gave minimum days to first floret opening during both seasons.

While, plants grown without nitrogen and humic acid (control) application had delayed sprouting the spike (95.66 and 99.00 day) and took more number of days to first floret opening (105.33 and 109.33 day).

The highest number of flowers per spike (11.00) were recorded with the application of $200 \mathrm{~kg} \mathrm{~N} \mathrm{fed}^{-1}$ with $350 \mathrm{mgl}^{-1}$ humic acid followed by $300 \mathrm{~kg} \mathrm{~N}^{-1} \mathrm{~d}^{-1}$ with $350 \mathrm{mg} \mathrm{l}^{-1}$ humic acid (11.00) in the first season. In the 
El-Kot et al. | SINAI Journal of Applied Sciences 9 (1) 2020 029-040

Table 3. Effect of nitrogen fertilizer levels and foliar spray with humic acid combinations on vegetative growth parameters of Gladiolus grandiflorus during 2017/2018 and 2018/2019 seasons

\begin{tabular}{|c|c|c|c|c|c|c|c|c|c|}
\hline \multirow{2}{*}{$\begin{array}{c}\begin{array}{c}\text { Nitrogen } \\
\text { fertilizer }\end{array} \\
\text { levels } \\
\left(\text { Kg fed }^{-1}\right)\end{array}$} & \multicolumn{9}{|c|}{ Vegetative growth parameters } \\
\hline & $\begin{array}{l}\text { Humic acid } \\
\text { rate }\left(\mathrm{mgl}^{-1}\right)\end{array}$ & $\begin{array}{c}\text { Number of } \\
\text { leaves }\end{array}$ & $\begin{array}{c}\text { Plant } \\
\text { height } \\
\text { (cm) }\end{array}$ & $\begin{array}{c}\text { Spike } \\
\text { length } \\
(\mathrm{cm})\end{array}$ & $\begin{array}{c}\text { Rachis } \\
\text { length } \\
\text { (cm) }\end{array}$ & $\begin{array}{c}\text { Leaf fresh } \\
\text { weight } \\
\text { (g) }\end{array}$ & $\begin{array}{c}\text { Leaf dry } \\
\text { weight (g) }\end{array}$ & $\begin{array}{c}\text { Weight of } \\
\text { spike } \\
\text { (g) }\end{array}$ & $\begin{array}{c}\text { Diameter of } \\
\text { spike } \\
(\mathrm{mm})\end{array}$ \\
\hline \multirow{7}{*}{ Control } & \multicolumn{9}{|c|}{ First Season 2017- 2018} \\
\hline & control & $8.00 \mathrm{a}$ & $66.00 \mathrm{~d}$ & $37.00 \mathrm{f}$ & $21.83 \mathrm{e}$ & $8.46 \mathrm{~d}$ & $3.53 \mathrm{~d}$ & $0.070 \mathrm{a}$ & $6.00 \mathrm{c}$ \\
\hline & 150 & $8.33 \mathrm{a}$ & $66.66 \mathrm{~d}$ & 42 ef & $22.33 \mathrm{e}$ & $8.52 \mathrm{~d}$ & $3.58 \mathrm{~cd}$ & $0.019 \mathrm{a}$ & $6.00 \mathrm{c}$ \\
\hline & 250 & $8.33 \mathrm{a}$ & $67.00 \mathrm{~d}$ & $47.16 \mathrm{de}$ & $24.33 \mathrm{de}$ & $8.91 \mathrm{~cd}$ & $3.86 \mathrm{~b}-\mathrm{d}$ & $0.023 \mathrm{a}$ & $6.3 \mathrm{c}$ \\
\hline & 350 & $8.66 \mathrm{a}$ & $71.33 \mathrm{~cd}$ & $54.66 \mathrm{~cd}$ & $24.50 \mathrm{de}$ & $9.42 \mathrm{~cd}$ & $3.91 \mathrm{~b}-\mathrm{d}$ & $0.028 \mathrm{a}$ & $6.3 \mathrm{c}$ \\
\hline & Control & $8.33 \mathrm{a}$ & $75.33 \mathrm{c}$ & $61.33 \mathrm{bc}$ & $26.50 \mathrm{c}-\mathrm{e}$ & $10.59 \mathrm{~b}-\mathrm{d}$ & $4.57 \mathrm{a}-\mathrm{d}$ & $0.026 \mathrm{a}$ & $6.3 \mathrm{c}$ \\
\hline & 150 & $8.66 \mathrm{a}$ & $76.33 \mathrm{c}$ & $61.66 \mathrm{bc}$ & $27.00 \mathrm{c}-\mathrm{e}$ & $10.62 \mathrm{~b}-\mathrm{d}$ & $5.05 \mathrm{a}-\mathrm{d}$ & $0.030 \mathrm{a}$ & $7.3 \mathrm{a}-\mathrm{c}$ \\
\hline \multirow[t]{2}{*}{$100 \mathrm{~kg} \mathrm{~N}$ fed $^{-1}$} & 250 & $8.66 \mathrm{a}$ & $84.00 \mathrm{c}$ & $62.66 \mathrm{a}-\mathrm{c}$ & $27.00 \mathrm{c}-\mathrm{e}$ & $10.87 \mathrm{~b}-\mathrm{d}$ & 5.05 a-d & $0.030 \mathrm{a}$ & $7.3 \mathrm{a}-\mathrm{c}$ \\
\hline & 350 & $9.00 \mathrm{a}$ & $84.33 \mathrm{~b}$ & $63.00 \mathrm{a}-\mathrm{c}$ & $27.83 \mathrm{c}-\mathrm{e}$ & $11.00 \mathrm{~b}-\mathrm{d}$ & $5.07 \mathrm{a}-\mathrm{d}$ & $0.031 \mathrm{a}$ & $7.3 \mathrm{a}-\mathrm{c}$ \\
\hline \multirow{5}{*}{$200 \mathrm{~kg} \mathrm{Nfed}^{-1}$} & Control & $8.66 \mathrm{a}$ & $85.66 \mathrm{ab}$ & $67.33 \mathrm{ab}$ & $33.33 \mathrm{a}-\mathrm{c}$ & $13.91 \mathrm{ab}$ & $6.07 \mathrm{a}-\mathrm{c}$ & $0.017 \mathrm{a}$ & $7.6 \mathrm{a}-\mathrm{c}$ \\
\hline & 150 & $8.66 \mathrm{a}$ & $90.66 \mathrm{ab}$ & $69.33 \mathrm{ab}$ & $33.33 \mathrm{a}-\mathrm{c}$ & $13.41 \mathrm{ab}$ & $6.21 \mathrm{ab}$ & $0.038 \mathrm{a}$ & $7.6 \mathrm{a}-\mathrm{c}$ \\
\hline & 250 & $9.33 \mathrm{a}$ & $91.00 \mathrm{a}$ & $70.16 \mathrm{ab}$ & $36.83 \mathrm{ab}$ & $13.52 \mathrm{ab}$ & $6.26 \mathrm{ab}$ & $0.043 \mathrm{a}$ & $8.6 \mathrm{ab}$ \\
\hline & 350 & $9.33 \mathrm{a}$ & $92.00 \mathrm{a}$ & $70.75 \mathrm{a}$ & $40.33 \mathrm{a}$ & $14.80 \mathrm{a}$ & $6.54 \mathrm{a}$ & $0.046 \mathrm{a}$ & $9.00 \mathrm{a}$ \\
\hline & control & $8.33 \mathrm{a}$ & $84.00 \mathrm{~b}$ & $64.33 \mathrm{ab}$ & $30.00 \mathrm{~b}-\mathrm{e}$ & $12.03 \mathrm{a}-\mathrm{c}$ & $4.45 \mathrm{a}-\mathrm{d}$ & $0.12 \mathrm{a}$ & $6.6 \mathrm{c}$ \\
\hline \multirow{3}{*}{$300 \mathrm{~kg} \mathrm{~N}$ fed $^{-1}$} & 150 & $8.33 \mathrm{a}$ & $84.00 \mathrm{~b}$ & $64.66 \mathrm{ab}$ & $30.00 \mathrm{~b}-\mathrm{e}$ & $12.30 \mathrm{a}-\mathrm{c}$ & $5.45 \mathrm{a}-\mathrm{d}$ & $0.13 \mathrm{a}$ & $7.00 \mathrm{bc}$ \\
\hline & 250 & $8.33 \mathrm{a}$ & $84.00 \mathrm{~b}$ & $65.16 \mathrm{ab}$ & $32.00 \mathrm{~b}-\mathrm{d}$ & $12.16 \mathrm{a}-\mathrm{c}$ & 5.46 a-d & $0.031 \mathrm{a}$ & $7.00 \mathrm{bc}$ \\
\hline & 350 & $9.00 \mathrm{a}$ & $84.33 \mathrm{~b}$ & $67.33 \mathrm{ab}$ & $34.16 \mathrm{a}-\mathrm{c}$ & $12.26 \mathrm{a}-\mathrm{c}$ & $5.71 \mathrm{a}-\mathrm{d}$ & $0.035 \mathrm{a}$ & $7.6 \mathrm{a}-\mathrm{c}$ \\
\hline \multicolumn{10}{|c|}{ Second Season 2018- 2019} \\
\hline \multirow{5}{*}{ Control } & Control & $7.66 \mathrm{~b}$ & $68.66 \mathrm{~d}$ & $55.00 \mathrm{~b}$ & $21.00 \mathrm{f}$ & $7.65 \mathrm{f}$ & $1.91 \mathrm{e}$ & $21.33 \mathrm{e}$ & $3.40 \mathrm{c}$ \\
\hline & 150 & $8.66 \mathrm{ab}$ & $69.66 \mathrm{~d}$ & $55.83 \mathrm{~b}$ & 21.50 ef & $9.14 \mathrm{ef}$ & $1.91 \mathrm{e}$ & $25.66 \mathrm{de}$ & $3.45 \mathrm{bc}$ \\
\hline & 250 & $9.00 \mathrm{ab}$ & $72.00 \mathrm{~cd}$ & $56.66 \mathrm{~b}$ & $22.33 \mathrm{ef}$ & $9.83 \mathrm{ef}$ & $2.07 \mathrm{e}$ & $26.00 \mathrm{de}$ & $3.64 \mathrm{a}-\mathrm{c}$ \\
\hline & 350 & $9.00 \mathrm{ab}$ & $75.33 \mathrm{~b}-\mathrm{d}$ & $57.00 \mathrm{~b}$ & $21.83 \mathrm{ef}$ & $9.83 \mathrm{ef}$ & $2.55 \mathrm{de}$ & $27.00 \mathrm{de}$ & $3.90 \mathrm{a}-\mathrm{c}$ \\
\hline & Control & $9.33 \mathrm{a}$ & $89.33 \mathrm{ab}$ & $62.00 \mathrm{ab}$ & $26.00 \mathrm{df}$ & $10.50 \mathrm{df}$ & $3.08 \mathrm{c}-\mathrm{e}$ & $30.00 \mathrm{ce}$ & $4.27 \mathrm{a}-\mathrm{c}$ \\
\hline \multirow{3}{*}{$100 \mathrm{~kg} \mathrm{~N} \mathrm{fed}^{-1}$} & 150 & $9.33 \mathrm{a}$ & $92.00 \mathrm{a}$ & $62.00 \mathrm{ab}$ & $32.00 \mathrm{a}-\mathrm{c}$ & $11.21 \mathrm{c}-\mathrm{e}$ & $6.27 \mathrm{a}$ & $39.50 \mathrm{a}-\mathrm{c}$ & $5.13 \mathrm{a}-\mathrm{c}$ \\
\hline & 250 & $9.33 \mathrm{a}$ & $92.00 \mathrm{a}$ & $73.33 \mathrm{a}$ & $33.33 \mathrm{ab}$ & $11.84 \mathrm{c}-\mathrm{e}$ & $6.43 \mathrm{a}$ & $41.00 \mathrm{a}-\mathrm{c}$ & $5.18 \mathrm{a}-\mathrm{c}$ \\
\hline & 350 & $9.33 \mathrm{a}$ & $92.33 \mathrm{a}$ & $73.33 \mathrm{a}$ & $32.83 \mathrm{ab}$ & $11.95 \mathrm{c}-\mathrm{e}$ & $6.50 \mathrm{a}$ & $45.00 \mathrm{ab}$ & $5.35 \mathrm{ab}$ \\
\hline \multirow{5}{*}{$200 \mathrm{~kg} \mathrm{~N} \mathrm{fed}^{-1}$} & Control & $9.00 \mathrm{ab}$ & $85.33 \mathrm{a}-\mathrm{c}$ & $69.50 \mathrm{a}$ & $31.33 \mathrm{a}-\mathrm{c}$ & 13.94 b-d & $4.47 \mathrm{bc}$ & $34.00 \mathrm{~b}-\mathrm{d}$ & $5.16 \mathrm{a}-\mathrm{c}$ \\
\hline & 150 & $9.00 \mathrm{ab}$ & 94.66 a & $69.83 \mathrm{a}$ & $31.33 \mathrm{a}-\mathrm{c}$ & $14.09 \mathrm{bc}$ & $5.68 \mathrm{ab}$ & $43.00 \mathrm{ab}$ & $5.35 \mathrm{ab}$ \\
\hline & 250 & $9.00 \mathrm{ab}$ & $95.00 \mathrm{a}$ & $72.00 \mathrm{a}$ & $33.33 \mathrm{ab}$ & $17.03 \mathrm{ab}$ & $5.90 \mathrm{a}$ & $44.00 \mathrm{ab}$ & $5.36 \mathrm{ab}$ \\
\hline & 350 & $9.00 \mathrm{ab}$ & 95.33 a & $74.33 \mathrm{a}$ & $36.83 \mathrm{a}$ & $17.32 \mathrm{a}$ & $7.12 \mathrm{a}$ & $47.00 \mathrm{a}$ & $5.38 \mathrm{a}$ \\
\hline & Control & $9.00 \mathrm{ab}$ & $82.00 \mathrm{a}-\mathrm{d}$ & $64.33 \mathrm{ab}$ & $27.00 \mathrm{c}-\mathrm{f}$ & $10.94 \mathrm{ab}$ & $3.65 \mathrm{~cd}$ & $33.33 \mathrm{~b}-\mathrm{d}$ & $4.78 \mathrm{a}-\mathrm{c}$ \\
\hline \multirow{3}{*}{$300 \mathrm{~kg} \mathrm{~N}$ fed $^{-1}$} & 150 & $9.00 \mathrm{ab}$ & 82.66 a-d & $65.33 \mathrm{ab}$ & $27.83 \mathrm{c}-\mathrm{f}$ & $11.08 \mathrm{c}-\mathrm{f}$ & $3.71 \mathrm{~cd}$ & $33.33 \mathrm{~b}-\mathrm{d}$ & $4.82 \mathrm{a}-\mathrm{c}$ \\
\hline & 250 & $9.00 \mathrm{ab}$ & $84.33 \mathrm{a}-\mathrm{c}$ & $66.66 \mathrm{ab}$ & $28.00 \mathrm{c}-\mathrm{f}$ & $11.16 \mathrm{c}-\mathrm{e}$ & $4.21 \mathrm{c}$ & $34.33 \mathrm{~b}-\mathrm{d}$ & $4.83 \mathrm{a}-\mathrm{c}$ \\
\hline & 350 & $9.00 \mathrm{ab}$ & $84.66 \mathrm{a}-\mathrm{c}$ & $67.33 \mathrm{ab}$ & $28.50 \mathrm{c}-\mathrm{e}$ & $11.21 \mathrm{c}-\mathrm{e}$ & $6.67 \mathrm{a}$ & $34.33 \mathrm{~b}-\mathrm{d}$ & $4.85 \mathrm{a}-\mathrm{c}$ \\
\hline
\end{tabular}

* Means followed by the same letter within each column are not significantly different at 0.05 level of probability according to Duncan's multiple range test. 
second season the application $200 \mathrm{~kg} \mathrm{~N}$ fed $^{-1}$ with all rates of humic acid gave the highest number of flowers (11.00) and there were no significant differences among these treatments. While, the control treatment gave the lowest number of flowers $(6.00$ and 7.00 in the two consecutive seasons, respectively).

Plants fertilized with $200 \mathrm{~kg} \mathrm{~N}^{-1} \mathrm{f}^{-1}$ and sprayed by $350 \mathrm{mg} \mathrm{l}^{-1}$ humic acid followed by $200 \mathrm{~kg} \mathrm{~N}_{\text {fed }}{ }^{-1}$ with $250 \mathrm{mg} \mathrm{l}^{-1}$ humic acid recorded the longest vase life (19.00, 19.00 and 20.33, 20.33 day) and water uptake $\left(93.00,93.00\right.$ and $\left.93.66,91.00 \mathrm{~cm}^{3}\right)$ in both seasons, respectively as compared with control treatment. These results are in line with Singh et al. (2001) who observed that gladiolus plants receiving higher rates of nitrogen $\left(30 \mathrm{~g} / \mathrm{m}^{2}\right)$ in a form of urea produced the maximum number of florets and diameter of florets. Also, Baral et al. (2012) on gladiolus found that plants received nitrogen up to $200 \mathrm{~kg} /$ ha gave the longest spike with more number of florets /spike. Moreover, largest spike and longest rachis produced by $300 \mathrm{~kg} / \mathrm{ha}$. In addition, El-Bably (2017) on tuberose stated that plants received $9 \mathrm{ml} / 1$ humic acid with soil drench method gave a significant increase in flowering parameters (spike and rachis length and number of florets per spike).

\section{Corms and Cormels Characteristics}

Table (5) shows the effect of nitrogen fertilization in a form of urea in combination with humic acid rate on corms and cormels characteristics.

Results revealed that significant variation were observed in weight of corms and weight of cormels, the heaviest corms and cormels were obtained with the application of $200 \mathrm{~kg}$ nitrogen $\mathrm{fed}^{-1}$ with $350 \mathrm{ppm}$ humic acid in both seasons, respectively. In addition, diameter of corms $(6.14$ and $5.38 \mathrm{~mm})$ and weight of cormels
(18.75 and $17.53 \mathrm{~g}$ ) were noticed with the same treatment. However, non-significant variation was observed in the treatment of $200 \mathrm{~kg}$ nitrogen $\mathrm{fed}^{-1}$ with the three rates of humic acid regard the weight of corms and No. of cormels in plants in the first season.

In the second season, there is no significant differences were showed in the weight of corms among all treatments. The maximum numbers of cormels were obtained in the treatment $200 \mathrm{~kg} \mathrm{~N}^{-1}$ in combination with $350 \mathrm{mg} \mathrm{l}^{-1}$ (31.33).

These results are in agreement with those of Bashir et al. (2016) they found that the high level of humic acid $(3.0 \mathrm{ml})$ in combination with NPK produced maximum cormels/plant, diameter of cormels and weight of cormels per plant than all other treatments. Also, Ghasemi et al. (2016) on tuberose, they observed that plants treated with $5 \mathrm{~kg} / \mathrm{ha}$ humic acid increased main bulbs weight and total bulbs weight.

\section{Chemical Constituents}

Table (6) reveals the effect of nitrogen fertilization and foliar spray with humic acid on chemical constituents of Gladiolus. Results indicated that a significant difference were observed on $\mathrm{N}, \mathrm{P}$ and $\mathrm{K}$ content in leaves. Also, the highest content of $\mathrm{N}$ (2.86 and 2.71\%), P (0.32 and 0.36) and $\mathrm{K}$ (3.23 and 3.16) were obtained under the application of $200 \mathrm{~kg}$ nitrogen $\mathrm{fed}^{-1}$ in combination with $350 \mathrm{mg}^{-1}$ humic acid during both seasons, these results are in agreement with those found by Abd-All and El-Namas (2017) who reported significant increase in total nitrogen, potassium and phosphorous of sweet potato plant receiving $4 \mathrm{~kg} /$ fed humic acid alone or in combination with potassium silicate as a foliar application., El-Bably (2017) on tuberose, stated that plants received $9 \mathrm{ml} / 1$ humic acid with soil drench method gave a significant increase in nitrogen, phosphorus and potassium percentage in leaves compared to untreated plants. 
Table 4. Effect of nitrogen fertilizer levels and foliar spray with humic acid combinations on flowering characteristics of Gladiolus grandiflorus during $2017 / 2018$ and $2018 / 2019$ seasons.

\begin{tabular}{|c|c|c|c|c|c|c|}
\hline $\begin{array}{c}\text { Nitrogen fertilizer } \\
\text { levels } \\
\left(\mathrm{Kg} \mathrm{Fed}^{-1}\right)\end{array}$ & $\begin{array}{c}\text { Humic acid } \\
\text { rate } \\
\left(\mathrm{mgl}^{-1}\right)\end{array}$ & $\begin{array}{l}\text { Days to spike } \\
\text { emergence }\end{array}$ & $\begin{array}{l}\text { Days to floret } \\
\text { opening }\end{array}$ & $\begin{array}{l}\text { No. of flowers } \\
\text { per spike }\end{array}$ & $\begin{array}{c}\text { Vase life } \\
\text { day }\end{array}$ & $\begin{array}{c}\text { Water } \\
\text { uptake } \\
\mathrm{Cm}^{3}\end{array}$ \\
\hline \multirow{6}{*}{ Control } & \multicolumn{6}{|c|}{ First Season 2017- 2018} \\
\hline & Control & $95.66 \mathrm{a}$ & $105.33 \mathrm{a}$ & $6.00 \mathrm{e}$ & $9.66 \mathrm{e}$ & $59.66 \mathrm{gh}$ \\
\hline & 150 & $95.00 \mathrm{a}$ & $103.00 \mathrm{ab}$ & $6.66 \mathrm{de}$ & $12.33 \mathrm{de}$ & $66.66 \mathrm{f}-\mathrm{h}$ \\
\hline & 250 & $92.66 \mathrm{ab}$ & $102.66 \mathrm{a}-\mathrm{c}$ & $7.66 \mathrm{c}-\mathrm{e}$ & $12.33 \mathrm{de}$ & $89.66 \mathrm{ac}$ \\
\hline & 350 & $82.50 \quad \mathrm{c}-\mathrm{e}$ & $101.66 \mathrm{a}-\mathrm{d}$ & $8.00 \mathrm{~b}-\mathrm{e}$ & $12.66 \mathrm{de}$ & $91.00 \mathrm{ab}$ \\
\hline & Control & 84.66 & $93.66 \mathrm{ef}$ & $7.66 \mathrm{c}-\mathrm{e}$ & $14.33 \mathrm{~cd}$ & $76.00 \mathrm{~d}-\mathrm{f}$ \\
\hline \multirow{3}{*}{$100 \mathrm{~kg} \mathrm{~N}$ fed $^{-1}$} & 150 & $86.33 \mathrm{c}-\mathrm{e}$ & $94.00 \mathrm{ef}$ & $9.00 \mathrm{a}-\mathrm{d}$ & $14.33 \mathrm{~cd}$ & $78.33 \mathrm{c}-\mathrm{f}$ \\
\hline & 250 & $87.33 \mathrm{bd}$ & $98.00 \mathrm{~b}-\mathrm{f}$ & $9.00 a-d$ & $14.66 \mathrm{~b}-\mathrm{d}$ & 81.66 a-e \\
\hline & 350 & $88.00 \mathrm{bc}$ & $99.33 \mathrm{~b}-\mathrm{e}$ & $9.33 \mathrm{a}-\mathrm{c}$ & $15.33 \mathrm{a}-\mathrm{d}$ & $85.00 \mathrm{ad}$ \\
\hline \multirow{3}{*}{$200 \mathrm{~kg} \mathrm{Nfed}^{-1}$} & Control & $80.46 \mathrm{e}$ & $92.33 \mathrm{f}$ & $10.00 \mathrm{a}-\mathrm{c}$ & $17.00 \mathrm{a}-\mathrm{c}$ & $56.00 \mathrm{~h}$ \\
\hline & 150 & $81.00 \mathrm{de}$ & $92.33 \mathrm{f}$ & $10.33 \mathrm{ab}$ & $18.66 \mathrm{a}$ & $58.33 \mathrm{gh}$ \\
\hline & 250 & $81.33 \mathrm{de}$ & $94.66 \mathrm{ef}$ & $11.00 \mathrm{a}$ & $19.00 \mathrm{a}$ & $93.00 \mathrm{a}$ \\
\hline \multirow{6}{*}{$300 \mathrm{~kg} \mathrm{~N}$ fed $^{-1}$} & 350 & $82.50 \mathrm{c}-\mathrm{e}$ & $96.66 \mathrm{ef}$ & $11.00 \mathrm{a}$ & $19.00 \mathrm{a}$ & $93.00 \mathrm{a}$ \\
\hline & Control & $84.00 \mathrm{c}-\mathrm{e}$ & $96.33 \mathrm{~d}-\mathrm{f}$ & $10.00 \mathrm{a}-\mathrm{c}$ & $15.33 \mathrm{a}-\mathrm{d}$ & $76.00 \mathrm{~d}-\mathrm{f}$ \\
\hline & 150 & $84.50 \mathrm{c}-\mathrm{e}$ & $97.33 \mathrm{~b}-\mathrm{f}$ & $10.33 \mathrm{ab}$ & $15.66 \mathrm{a}-\mathrm{d}$ & $76.66 \mathrm{~d}-\mathrm{f}$ \\
\hline & 250 & $84.66 \mathrm{c}-\mathrm{e}$ & $98.00 \mathrm{~b}-\mathrm{f}$ & $10.33 \mathrm{ab}$ & $18.00 \mathrm{a}-\mathrm{c}$ & $68.33 \mathrm{e}-\mathrm{g}$ \\
\hline & 350 & $85.00 \mathrm{c}-\mathrm{e}$ & $98.66 \mathrm{~b}-\mathrm{e}$ & $11.00 \mathrm{a}$ & $18.33 \mathrm{ab}$ & $79.33 \mathrm{~b}-\mathrm{e}$ \\
\hline & \multicolumn{6}{|c|}{ Second Season 2018- 2019} \\
\hline \multirow{4}{*}{ Control } & Control & $99.00 \mathrm{a}$ & $109.33 \mathrm{a}$ & $7.33 \mathrm{~b}$ & $12.00 \mathrm{~d}$ & $24.00 \mathrm{f}$ \\
\hline & 150 & $98.33 \mathrm{ab}$ & $109.00 \mathrm{a}$ & $8.33 \mathrm{ab}$ & $13.66 \mathrm{~cd}$ & $51.66 \mathrm{e}$ \\
\hline & 250 & $97.00 \quad a-c$ & $107.66 \mathrm{a}$ & $8.33 \mathrm{ab}$ & $13.66 \mathrm{~cd}$ & $53.33 \mathrm{~d}-\mathrm{e}$ \\
\hline & 350 & 96.66 a-c & $105.33 \mathrm{ab}$ & $8.66 \mathrm{ab}$ & $14.33 \mathrm{~cd}$ & $58.33 \mathrm{c}-\mathrm{e}$ \\
\hline \multirow{4}{*}{$100 \mathrm{~kg} \mathrm{~N}$ fed $^{-1}$} & Control & $91.00 \mathrm{~d}-\mathrm{e}$ & $101.66 \mathrm{a}-\mathrm{c}$ & $9.33 \mathrm{ab}$ & $15.33 \mathrm{~b}-\mathrm{d}$ & $69.33 \mathrm{~b}-\mathrm{e}$ \\
\hline & 150 & $92.00 \mathrm{~b}-\mathrm{d}$ & $102.00 \mathrm{a}-\mathrm{c}$ & $9.33 \mathrm{ab}$ & $16.66 \mathrm{a}-\mathrm{c}$ & $70.00 \mathrm{~b}-\mathrm{e}$ \\
\hline & 250 & $93.00 \mathrm{a}-\mathrm{c}$ & $103.00 \mathrm{a}-\mathrm{c}$ & $9.33 \mathrm{ab}$ & $16.66 \mathrm{a}-\mathrm{c}$ & $71.66 \mathrm{a}-\mathrm{d}$ \\
\hline & 350 & $93.66 \mathrm{a}-\mathrm{c}$ & $103.33 \mathrm{a}-\mathrm{c}$ & $9.66 \mathrm{ab}$ & $17.00 \mathrm{a}-\mathrm{c}$ & $71.66 \mathrm{a}-\mathrm{d}$ \\
\hline \multirow{4}{*}{$200 \mathrm{~kg} \mathrm{~N}$ fed $^{-1}$} & Control & $79.00 \mathrm{f}$ & $95.66 \mathrm{c}$ & $11.00 \mathrm{a}$ & $19.66 \mathrm{a}$ & $84.33 \mathrm{ab}$ \\
\hline & 150 & $79.00 \mathrm{f}$ & $95.66 \mathrm{c}$ & $11.00 \mathrm{a}$ & $19.66 \mathrm{a}$ & $88.33 \mathrm{ab}$ \\
\hline & 250 & $80.00 \mathrm{f}$ & $95.66 \mathrm{c}$ & $11.33 \mathrm{a}$ & $20.33 \mathrm{a}$ & $90.66 \mathrm{a}$ \\
\hline & 350 & $80.33 \mathrm{f}$ & $96.33 \mathrm{c}$ & $11.33 \mathrm{a}$ & $20.33 \mathrm{a}$ & $91.00 \mathrm{a}$ \\
\hline \multirow{4}{*}{$300 \mathrm{~kg} \mathrm{~N}$ fed $^{-1}$} & Control & $83.33 \mathrm{f}$ & $98.33 \mathrm{bc}$ & $10.00 \mathrm{ab}$ & $19.00 \mathrm{ab}$ & $75.00 \mathrm{a}-\mathrm{c}$ \\
\hline & 150 & $83.66 \mathrm{f}$ & $98.66 \mathrm{bc}$ & $10.00 \mathrm{ab}$ & $19.00 \mathrm{ab}$ & $76.66 \mathrm{a}-\mathrm{c}$ \\
\hline & 250 & $84.33 \mathrm{f}$ & $98.66 \mathrm{bc}$ & $10.33 \mathrm{ab}$ & $19.33 \mathrm{ab}$ & $77.00 \mathrm{a}-\mathrm{c}$ \\
\hline & 350 & 85.33 ef & $99.00 \mathrm{bc}$ & $10.33 \mathrm{ab}$ & $19.33 \mathrm{ab}$ & $77.33 \mathrm{a}-\mathrm{c}$ \\
\hline
\end{tabular}

* Means followed by the same letter within each column are not significantly different at 0.05 level of probability according to Duncan's multiple range test. 
Table 5. Effect of nitrogen fertilizer levels and foliar spray with humic acid combinations on corms and cormels charachteristics of Gladiolus grandiflorus during 2017/2018 and 2018/2019 seasons.

\begin{tabular}{|c|c|c|c|c|c|}
\hline \multirow[b]{2}{*}{$\begin{array}{l}\text { Nitrogen fertilizer } \\
\text { Level }\left(\mathrm{Kg} \mathrm{fed}^{-1}\right)\end{array}$} & \multicolumn{5}{|c|}{ Corms charachteristics } \\
\hline & $\begin{array}{l}\text { Humic acid } \\
\text { rate }\left(\mathrm{mgl}^{-1}\right)\end{array}$ & $\begin{array}{c}\text { Weight of } \\
\text { corms } \\
(\mathrm{g}) \\
\end{array}$ & $\begin{array}{l}\text { Diameter of corms } \\
(\mathbf{m m})\end{array}$ & Number of cormels & $\begin{array}{l}\text { Weight of cormels } \\
\text { (g) }\end{array}$ \\
\hline \multirow{5}{*}{ Control } & \multicolumn{5}{|c|}{ First Season 2017- 2018} \\
\hline & Control & $25.95 \mathrm{~d}$ & $3.77 \mathrm{~d}$ & $7.33 \mathrm{~b}$ & $8.22 \mathrm{ab}$ \\
\hline & 150 & $27.90 \mathrm{~cd}$ & $4.08 \mathrm{~cd}$ & $8.33 \mathrm{~b}$ & $9.84 \mathrm{ab}$ \\
\hline & 250 & $31.99 \mathrm{~b}-\mathrm{d}$ & $4.72 \mathrm{a}-\mathrm{d}$ & $9.00 \mathrm{~b}$ & $14.33 \mathrm{ab}$ \\
\hline & 350 & $32.98 \mathrm{~b}-\mathrm{d}$ & $5.07 \mathrm{ad}$ & $11.00 \mathrm{~b}$ & $19.66 \mathrm{a}$ \\
\hline \multirow{4}{*}{$100 \mathrm{~kg} \mathrm{~N}$ fed $^{-1}$} & Control & $46.20 \mathrm{ab}$ & $4.53 \mathrm{e}-\mathrm{d}$ & $16.00 \mathrm{ab}$ & $5.56 \mathrm{~b}$ \\
\hline & 150 & $46.55 \mathrm{ab}$ & $4.96 \mathrm{a}-\mathrm{d}$ & $16.66 \mathrm{ab}$ & $7.67 \mathrm{ab}$ \\
\hline & 250 & $44.87 \mathrm{a}-\mathrm{c}$ & $5.08 \mathrm{a}-\mathrm{d}$ & $17.33 \mathrm{ab}$ & $7.71 \mathrm{ab}$ \\
\hline & 350 & $52.30 \mathrm{a}$ & $5.73 \mathrm{ab}$ & $17.33 \mathrm{ab}$ & $14.04 \mathrm{ab}$ \\
\hline \multirow{4}{*}{$200 \mathrm{~kg} \mathrm{Nfed}^{-1}$} & Control & $48.67 \mathrm{ab}$ & $5.22 \mathrm{a}-\mathrm{d}$ & $21.00 \mathrm{ab}$ & $5.24 \mathrm{~b}$ \\
\hline & 150 & $56.69 \mathrm{a}$ & $5.27 \mathrm{a}-\mathrm{d}$ & $29.00 \mathrm{a}$ & $8.77 \mathrm{ab}$ \\
\hline & 250 & $56.82 \mathrm{a}$ & $5.49 \mathrm{a}-\mathrm{c}$ & $29.33 \mathrm{a}$ & $10.09 \mathrm{ab}$ \\
\hline & 350 & $59.08 \mathrm{a}$ & $6.14 \mathrm{a}$ & $31.33 \mathrm{a}$ & $18.75 \mathrm{a}$ \\
\hline \multirow{4}{*}{$300 \mathrm{~kg} \mathrm{~N}$ fed $^{-1}$} & Control & $47.30 \mathrm{ab}$ & $4.12 \mathrm{~cd}$ & $21.00 \mathrm{ab}$ & $4.86 \mathrm{~b}$ \\
\hline & 150 & $48.04 \mathrm{ab}$ & $5.21 \mathrm{a}-\mathrm{d}$ & $21.00 \mathrm{ab}$ & $5.82 \mathrm{~b}$ \\
\hline & 250 & $50.40 \mathrm{ab}$ & $5.26 \mathrm{a}-\mathrm{d}$ & $23.33 \mathrm{a} \mathrm{b}$ & $11.19 \mathrm{ab}$ \\
\hline & 350 & $50.73 \mathrm{ab}$ & $5.13 \mathrm{a}-\mathrm{d}$ & $23.33 \mathrm{ab}$ & $11.61 \mathrm{ab}$ \\
\hline \multirow{9}{*}{$100 \mathrm{~kg} \mathrm{~N} \mathrm{fed}^{-1}$} & \multicolumn{5}{|c|}{ Second Season 2018- 2019} \\
\hline & Control & $28.42 \mathrm{a}$ & $3.40 \mathrm{c}$ & $5.33 \mathrm{c}$ & $3.66 \mathrm{e}$ \\
\hline & $150 \mathrm{mgl}$ & $28.91 \mathrm{a}$ & $3.45 \mathrm{bc}$ & $5.66 \mathrm{c}$ & $4.13 \mathrm{de}$ \\
\hline & 250 & $30.01 \mathrm{a}$ & $3.64 \mathrm{a}-\mathrm{c}$ & $6.66 \mathrm{bc}$ & $4.92 \mathrm{c}-\mathrm{e}$ \\
\hline & 350 & $30.32 \mathrm{a}$ & $3.90 \mathrm{a}-\mathrm{c}$ & $8.33 \mathrm{bc}$ & $6.68 \mathrm{~b}-\mathrm{e}$ \\
\hline & Control & $35.40 \mathrm{a}$ & $4.27 \mathrm{a}-\mathrm{c}$ & $14.00 \mathrm{a}-\mathrm{c}$ & 9.25 a-e \\
\hline & 150 & $35.83 a$ & $5.13 \mathrm{a}-\mathrm{c}$ & $15.66 \mathrm{a}-\mathrm{c}$ & 9.45 a-e \\
\hline & 250 & $49.05 \mathrm{a}$ & $5.18 \mathrm{a}-\mathrm{c}$ & $28.00 \mathrm{a}-\mathrm{c}$ & $14.45 \mathrm{a}-\mathrm{d}$ \\
\hline & 350 & $50.08 \mathrm{a}$ & $5.35 \mathrm{ab}$ & $33.66 \mathrm{a}-\mathrm{c}$ & $15.14 \mathrm{ab}$ \\
\hline \multirow{4}{*}{$200 \mathrm{~kg} \mathrm{~N}$ fed $^{-1}$} & Control & $51.08 \mathrm{a}$ & $5.16 \mathrm{a}-\mathrm{c}$ & $30.66 \mathrm{a}-\mathrm{c}$ & 13.83 a-e \\
\hline & 150 & $51.64 \mathrm{a}$ & $5.35 \mathrm{ab}$ & $31.00 \mathrm{a}-\mathrm{c}$ & 14.10 a-e \\
\hline & 250 & $51.71 \mathrm{a}$ & $5.36 \mathrm{ab}$ & $36.33 \mathrm{ab}$ & $15.18 \mathrm{a}-\mathrm{c}$ \\
\hline & 350 & $53.81 \mathrm{a}$ & $5.38 \mathrm{a}$ & $39.00 \mathrm{a}$ & $17.53 \mathrm{a}$ \\
\hline \multirow{4}{*}{$300 \mathrm{~kg} \mathrm{~N}$ fed $^{-1}$} & Control & $42.86 \mathrm{a}$ & $4.82 \mathrm{a}-\mathrm{c}$ & $21.66 \mathrm{a}-\mathrm{c}$ & 8.44 a-e \\
\hline & 150 & $43.21 \mathrm{a}$ & $4.83 \mathrm{a}-\mathrm{c}$ & $22.33 \mathrm{a}-\mathrm{c}$ & 9.14 a-e \\
\hline & 250 & $43.68 \mathrm{a}$ & $4.85 \mathrm{a}-\mathrm{c}$ & $26.33 \mathrm{a}-\mathrm{c}$ & $9.22 \mathrm{a}-\mathrm{e}$ \\
\hline & 350 & $45.91 \mathrm{a}$ & $4.78 \mathrm{a}-\mathrm{c}$ & $29.00 \mathrm{a}-\mathrm{c}$ & $12.66 \mathrm{a}-\mathrm{e}$ \\
\hline
\end{tabular}

* Means followed by the same letter within each column are not significantly different at 0.05 level of probability according to Duncan's multiple range test. 
Table 6. Effect of nitrogen fertilizer levels and foliar spray with humic acid combinations on chemical constituent of Gladiolus grandiflorus during 2017/ 2018 and $2018 / 2019$ seasons.

\begin{tabular}{|c|c|c|c|c|}
\hline \multirow{2}{*}{$\begin{array}{l}\text { Nitrogen fertilizer } \\
\text { level }\left(\mathrm{kg} \mathrm{fed}^{-1}\right)\end{array}$} & \multicolumn{4}{|c|}{ Chemical constituent } \\
\hline & $\begin{array}{l}\text { Humic acid rate } \\
\qquad\left(\mathrm{mgl}^{-1}\right)\end{array}$ & $\mathbf{N}(\%)$ & $\mathbf{P}(\%)$ & $\mathbf{K}(\%)$ \\
\hline & \multicolumn{4}{|c|}{ First Season 2016- 2017} \\
\hline \multirow{5}{*}{ Control } & Control & 0.92 o & $0.13 \mathrm{~d}$ & $1.13 \mathrm{p}$ \\
\hline & 150 & $0.94 \mathrm{n}$ & $0.13 \mathrm{~d}$ & $1.16 \mathrm{o}$ \\
\hline & 250 & $0.95 \mathrm{mn}$ & $0.14 \mathrm{~d}$ & $1.25 \mathrm{n}$ \\
\hline & 350 & $0.96 \mathrm{~m}$ & $0.15 \mathrm{~d}$ & $1.36 \mathrm{~m}$ \\
\hline & Control & 1.091 & $0.18 \mathrm{c}$ & 1.731 \\
\hline \multirow{3}{*}{$100 \mathrm{~kg} \mathrm{~N}$ fed $^{-1}$} & 150 & $1.21 \mathrm{k}$ & $0.19 \mathrm{c}$ & $1.75 \mathrm{k}$ \\
\hline & 250 & $1.32 \mathrm{j}$ & $0.19 \mathrm{c}$ & $1.87 \mathrm{j}$ \\
\hline & 350 & $1.41 \mathrm{i}$ & $0.20 \mathrm{c}$ & $1.98 \mathrm{i}$ \\
\hline \multirow{3}{*}{$200 \mathrm{~kg} \mathrm{Nfed}^{-1}$} & Control & $2.59 \mathrm{~d}$ & $0.29 \mathrm{a}$ & $2.96 \mathrm{~d}$ \\
\hline & 150 & $2.63 \mathrm{c}$ & $0.30 \mathrm{a}$ & $3.06 \mathrm{c}$ \\
\hline & 250 & $2.75 \mathrm{~b}$ & $0.31 \mathrm{a}$ & $3.15 \mathrm{~b}$ \\
\hline \multirow{5}{*}{$300 \mathrm{~kg} \mathrm{~N}$ fed $^{-1}$} & 350 & $2.86 \mathrm{a}$ & $0.32 \mathrm{a}$ & $3.23 \mathrm{a}$ \\
\hline & control & $1.80 \mathrm{~h}$ & $0.24 \mathrm{~b}$ & $2.35 \mathrm{~h}$ \\
\hline & 150 & $1.84 \mathrm{~g}$ & $0.24 \mathrm{~b}$ & $2.44 \mathrm{~g}$ \\
\hline & 250 & $1.95 \mathrm{f}$ & $0.25 \mathrm{~b}$ & $2.55 \mathrm{f}$ \\
\hline & 350 & $2.06 \mathrm{e}$ & $0.26 \mathrm{~b}$ & $2.65 \mathrm{e}$ \\
\hline & \multicolumn{4}{|c|}{ Second Season 2017- 2018} \\
\hline \multirow{4}{*}{ Control } & Control & $0.91 \mathrm{i}$ & $0.14 \mathrm{~h}$ & $1.05 \mathrm{f}$ \\
\hline & 150 & $0.92 \mathrm{hi}$ & $0.15 \mathrm{~h}$ & $1.07 \mathrm{f}$ \\
\hline & 250 & $0.93 \mathrm{hi}$ & $0.16 \mathrm{~g}$ & $1.16 \mathrm{f}$ \\
\hline & 350 & $0.95 \mathrm{hi}$ & $0.17 \mathrm{f}$ & $1.38 \mathrm{ef}$ \\
\hline \multirow{4}{*}{$100 \mathrm{~kg} \mathrm{~N}$ fed $^{-1}$} & Control & $1.33 \mathrm{f}$ & $0.33 \mathrm{~d}$ & $1.61 \mathrm{e}$ \\
\hline & 150 & $1.64 \mathrm{e}$ & $0.33 \mathrm{~d}$ & $1.66 \mathrm{e}$ \\
\hline & 250 & $1.06 \mathrm{gh}$ & $0.35 \mathrm{~b}$ & $1.76 \mathrm{e}$ \\
\hline & 350 & $1.17 \mathrm{gf}$ & $0.36 \mathrm{a}$ & $2.29 \mathrm{~d}$ \\
\hline \multirow{4}{*}{$200 \mathrm{~kg} \mathrm{~N}$ fed $^{-1}$} & Control & $2.47 \mathrm{c}$ & $0.33 \mathrm{c}$ & $2.65 \mathrm{~b}-\mathrm{d}$ \\
\hline & 150 & $2.49 \mathrm{bc}$ & $0.34 \mathrm{~d}$ & $2.77 \mathrm{a}-\mathrm{c}$ \\
\hline & 250 & $2.62 \mathrm{ab}$ & $0.35 \mathrm{~b}$ & $3.06 \mathrm{ab}$ \\
\hline & 350 & $2.71 \mathrm{a}$ & $0.36 \mathrm{a}$ & $3.16 \mathrm{a}$ \\
\hline \multirow{4}{*}{$300 \mathrm{~kg} \mathrm{~N}$ fed $^{-1}$} & Control & $1.63 \mathrm{~d}$ & $0.27 \mathrm{e}$ & $2.24 \mathrm{~d}$ \\
\hline & 150 & $1.64 \mathrm{~d}$ & $0.28 \mathrm{~d}$ & $2.34 \mathrm{~cd}$ \\
\hline & 250 & $1.74 \mathrm{de}$ & $0.28 \mathrm{~d}$ & $2.46 \mathrm{~cd}$ \\
\hline & 350 & $1.85 \mathrm{~d}$ & $0.28 \mathrm{~d}$ & $2.56 \mathrm{~cd}$ \\
\hline
\end{tabular}

*Means followed by the same letter within each column are not significantly different at 0.05 level of probability according to Duncan's multiple range test. 
Also, Fahmy and Hassan (2019) demonstrated that the combination between $100 \%$ of recommended dose with $4 \mathrm{l} /$ fed humic acid gave a significant effect for roselle chemical constituents $(\mathrm{N}, \mathrm{P}$ and $\mathrm{K})$ compared to control.

\section{Conclusion}

From above mentioned results, it is preferable to fertilized Gladiolus grandiflorus with $200 \mathrm{~kg}$ nitrogen $\mathrm{fed}^{-1}$ in a form of urea and spraying with $350 \mathrm{mg} \mathrm{l}^{-1}$ humic acid to enhance the vegetative growth, flowering characteristics, corms and cormels characteristics and chemical constituents.

\section{REFERENCES}

Abd-All, A. and A.E. El-Namas. (2017). Effect of humic acid and foliar application of different potassium sources on yield, quality and water use efficiency of sweet potato grown under drip irrigation in sandy soil. Alex. sci. Exchange J., 38 (3): 543-553.

Anserwadekar, K.W. and Patil, V.K. (1986). Vase-life studies of gladiolus (Gladiolus grandiflora) cv. H.B. Pitt 1Effect of NPK and spacing on vase life II. Effect of different chemicals. Acta Hort., 181: 279-283.

Baral, S.P.; D.R. Baral; D.M. Gautam and U.K. Pun (2012). Influence of nitrogen on growth performance, cut flower characteristics and corm/ cormel production of gladiolus. Nepal J. Sci. and Tech., 13(1): 25-31.

Bashir, M; R.W.K. Qadri; I. Khan; M. Zain; A. Rasool and U. Ashraf (2016). Humic acid application improves the growth floret and bulb indices of gladiolus. Paki J. Sci. 68(2): 121-127.

Baldoto, M.A. and L.E.B. Baldoto (2013). Gladiolus development in response to bulb treatment with different concentrations of humic acids. Rev. Ceres, Vicosa. 60 (1): 138-142.
Black, C.A. (1965). Methods of soil analysis. Part 2. Amer. Soci. Agric. $\{\mathrm{NC}\}$ publisher, Madison, Wisconsin.

Bose, T.K. and L.P. Yadav. (1989). Commercial flowers. Naya porkash, Calcutta-7, India. P. 267.

Chaudhary, N.; K. Swaroop; T. Janakiram; D.R. Biswas and G. Singh. (2013). Effect of integrated nutrient management on vegetative growth and flowering characters of Gladiolus. I J. Hort., 70 (1): 156-159.

Chen, Y. and T. Aviad (1990). Effects of humic substances in soil and crop Selected reading. SSSA and ASA, Madison, Wisconsin, USA. pp. 161-186.

Duncan, B.D. (1955). Multipe Ranges and Multiple F test. Biometrics, 11: 1-42.

El-Bably, S.M.Z. (2017). Effect of some natural material additives on growth and flowering of tuberose (Polianthes tuberosa, L.) bulbs. J. Plant Prod., 8 (9): 895-906

El-khateeb, M.A.; A.B. El-Attar and R.M. Nour (2017). Application of plant bio-stimulants to improve the biological response and essential oil productive of marjoram (Majorana hortensis) plants. Middle East J. Agric. Res., 6(4): 928941.

Fahmy, A.A. and H.M.S. Hassan (2019). Influence of different NPK fertilization levels and humic acid rates on growth, yield and chemical constituents of roselle (Hibiscus subdariffa L.). Middle East. J. Agric. Res., 8(4): 1182-1189.

Ghasemi, H.; M. Rezaei; H. Asghari; H. Ghorbani-Ghouzhdi and A. Sharififar (2016). cultivation of tuberose in pot and field with humic acid treatments under a semi-arid climate. Indian J. Hort. 73(3): 391-395.

Goldblatt, P. and J.C. Manning (1998). Gladiolus in Southern Africa: Systematics, 
Biology and Evaluation. Fernwood press, Crape Town., 464.

Haghighi, S.; T. Sakinejad and S.H. Lack (2011). Evalution of changes the equalization and quantitative yield of horse been (Vicia faba) plant in the level of humic acid fertilizer. Life Sci., 8 (3): 133-144.

Harper, J.E. and Paulsen, G.M. (1969). Nitrogen assimilation and protein synthesis in wheat seedling as affected by mineral nutrition. I. Macronutrients Plant Physiol., 69-75.

Jackson, M.L. (1967). Soil chemical analysis advanced course. Puble. Bythe auther. Dept. Soils, Univ. Wise., Madison 6, Wishensin, Univ.

Khalf-Allah, M.M. (2014). Flowers and Ornamental Plants (Flowering Bulbs). Kafr El-Sheikh Univ., 138.

Manning, J. and P. Goldblatt (2008). The iris family: Nature history and classification. Port land, Oregon. Timber press. 138-142.

Patra, S.K. and C.R. Mohanty (2015). Vase life study in different varieties of gladiolus. I. J. Agri. 5(2): 27-32.

Peterburgski, A.V. (1968). Handbook of Agronomic Chemistry. Kolas Publishing Hous, Moscow, (in Russian), 29-86.

Pregle, E. (1945). Quantitive Organic Micro-Analysis. $4^{\text {th }}$ Ed. J. Chudrial, London.
Singh, A.K.; N.P. Singh and V.B. Singh (2001). Growth, flowering and corm production in gladiolus c.v Oscar as influenced by nitrogen and spacing in acidic soil of Nagaland. Indian J. Hill Farmg. 14(1): 128-131.

Singh, M.F. and S.M. Ramasingh (1969). Response of onion to different fertilizer application. Indian J. Agric. Sci., 39 (10) 1026-1028.

\section{Sharif, M.; R.A. Khattar and M.S. Sarir} (2002). Effect of different levels of lignit-iccaal derived humic acid on growth of maiz plants. Communication in Soil Science and Plant Analysis., 33(1): 3567-3580.

Thana, P.P.S.; S. Earasilp; E. Pawelzik and S. Gorinstein (2008). Influence of various nitrogen application on protein and amino acid profiles of amaranth and quinoa. J. Agric. Food Chem., 56: 11464-11470.

Valdrighi, M.M.; A. Pear; M. Agnolucci; S. Frassinetti; D. Lunardi and G. Vallini (1996). Effect of compostderived humic acid on vegetable biomass production and microbial growth within a plant (Cichorium intubus) soil system: a comparative study. Agric. Ecosys.

Woltz, S.S. (1972). The world of the gladiolus, NaGC, USA, pp.143-149.

Patra, S.K. and C.R. Mohanty (2015). Vase life study in different varieties of gladiolus I.J. Agric., 5(2): 27-32. 


$$
\text { الملخص العربي }
$$

\section{تأثير مستوي التسميذ النيتروجيني ومعدل حامض الهيوميك علي إنتاجية الجلاديولس} هدير رمضان محمد القط، سونيا عطيه شحاته، هاني محمد سامي حسن ومحمد أحمد محمود علي

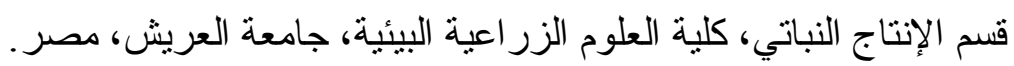

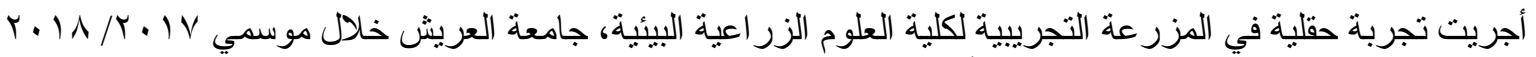

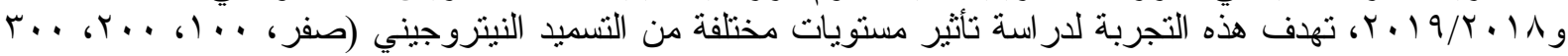

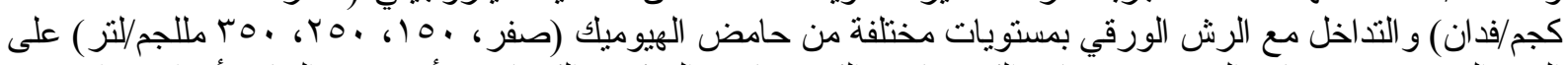

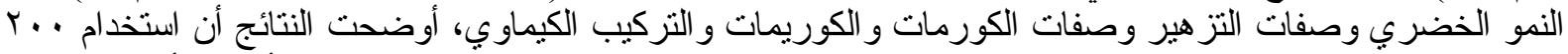

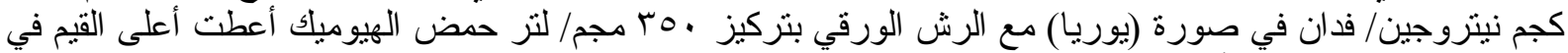

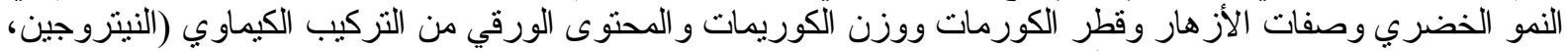

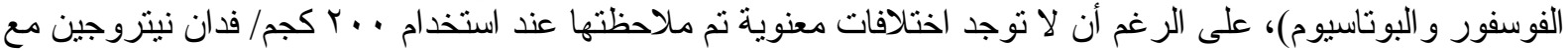

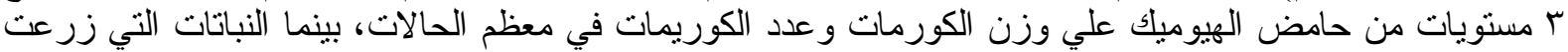

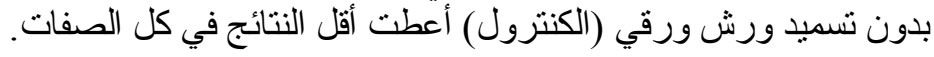
الكلمات الإسترشادية: النسميد النيتروجيني الكيماوي، رش رون حمض الهيوميك، الجلاديولس. 